\title{
Qualidade de vida e prognóstico nos carcinomas epidermóides de cabeça e pescoço
}

\author{
Quality of life and \\ proginosis of \\ squamous cell \\ carcinoma of the head \\ and neck
}

\author{
Ali Amar ${ }^{1}$, Abrão Rapoport ${ }^{1}$, Sérgio A. Franzi ${ }^{1}$, \\ Clarice Bisordi' ${ }^{2}$ Carlos N. Lehn
}

Palavras-chave: neoplasias de cabeça e pescoço, carcinoma epidermóide, qualidade de vida.

Key words: head and neck neoplasms, squamous cell carcinoma, quality of life.

Resumo / Summary

\begin{abstract}
O bjetivo: avaliar um questionário para mensuração da qualidade de vida e sua relação com o prognóstico em pacientes com câncer de cabeça e pescoço. Forma de estudo: prospectivo clínico. Material e Método: estudo de 31 pacientes com carcinoma epidermóide de vias aerodigestivas superiores atendidos no Departamento de Cirurgia de Cabeça e Pescoço e Otorrinolaringologia do Hospital Heliópolis, Hosphel, São Paulo, no período de agosto de 1999 a novembro de 2000. Na avaliação da qualidade de vida empregou-se o questionário QLQ-C30 e o módulo QLQH\&N35, ambos fornecidos pela EORTC, sendo realizada previamente ao tratamento. Foi avaliada a diferença de pontuação entre os pacientes que apresentavam doença controlada e aqueles com recidiva nos primeiros 12 meses após o tratamento. Resultados: foi observada uma diferença significativa na pontuação entre os pacientes assintomáticos e aqueles com recidiva da doença nas escalas relacionadas à fadiga, dificuldade de comer em público e qualidade de vida global $(p \leq 0,05)$. A pontuação obtida neste grupo de pacientes foi semelhante à observada em outras populações. Conclusão: a qualidade de vida prévia ao tratamento pode constituir um indicador prognóstico para os pacientes com câncer de cabeça e pescoço.
\end{abstract}

\begin{abstract}
A im: a quality life questionnaire (QLQ) assessment and its relationship with prognostic of patients with head and neck cancer. Study design: prospective clinical. Material and method: a study of 31 patients with squamous cell carcinoma of upper aerodigestive tract submitted to therapy in Head and Neck Surgery and Otorhinolaryngology Department of Heliópolis Hospital, Hosphel between August,1999 and November, 2000. For evaluation of quality of life it was used the questionnaire QLQ-C30 and the module QLQ-H\&N35, both supplied by EORTC, accomplished previously to the treatment. The differences of scores among patients with controlled disease and those with recurrence in the first 12 months after treatment was evaluated. Results: a significant difference was observed in scores between assimptomatic patients and those with recurrence, when related to the fadiga, problems of eating in public and global quality of life $(\mathrm{p} \leq 0,05)$. The scores in this group were similar to those observed in other populations. Conclusion: the pre-treatment quality of life can be a prognostic indicator for patients with head and neck cancer.
\end{abstract}

\footnotetext{
${ }^{1}$ Cirurgião do Departamento de Cirurgia de Cabeça e Pescoço e Otorrinolaringologia do Hospital Heliópolis, Hosphel, São Paulo.

${ }_{2}^{2}$ Psicóloga do Departamento de Cirurgia de Cabeça e Pescoço e Otorrinolaringologia do Hospital Heliópolis, Hosphel, São Paulo.
}

Trabalho realizado no Departamento de Cirurgia de Cabeça e Pescoço e Otorrinolaringologia do Hospital Heliópolis, Hosphel, São Paulo. Endereço para correspondência: Abrão Rapoport - Rua Iramaia, 136 Jd. Europa - 01450-020 - São Paulo SP

Tel. (0xx11) 273.8224 - E-mail: cpgcp.hosphel@attglobal.net

Artigo recebido em 28 de janeiro de 2002. Artigo aceito em 11 de abril de 2002 


\section{INTRODUÇÃO}

A avaliação da qualidade de vida tem ganho espaço crescente no tratamento dos pacientes com tumores de cabeça e pescoço, pois este geralmente causa significativo comprometimento estético e funcional. Com a prática dos protocolos de preservação de órgãos ou estruturas, a qualidade de vida despertou maior interesse, tornando-se um argumento para justificar modalidades terapêuticas menos mutiladoras quando os resultados oncológicos são equivalentes.

Diversos instrumentos foram criados com o objetivo de quantificar a qualidade de vida. Apesar do termo ter se popularizado, o conceito de qualidade de vida reflete aspectos emocionais, culturais e econômicos muito mais complexos do que os questionários desenvolvidos para quantificá-la. Em nosso meio, uma parcela significativa dos pacientes apresenta baixo nível de escolaridade e rendimentos insuficientes para atender às suas necessidades básicas. O efeito da doença no cotidiano destes pacientes e a utilidade dos instrumentos de avaliação da qualidade de vida ainda não foram definidos nesta população. A necessidade de tratar rapidamente o paciente com câncer faz com que a proposta terapêutica seja embasada quase que exclusivamente no estadiamento da doença, de acordo com esquemas previamente definidos, nem sempre levando em conta o benefício do procedimento segundo critérios referidos pelos pacientes (princípio bioético da maior relevância). Estes reagem de forma diferente às seqüelas do tratamento, como também podem valorizar diferentemente os seus sintomas. Não há relação entre alguns itens da avaliação da qualidade de vida e o estadiamento da doença, mas a qualidade de vida apresenta relação com o prognóstico ${ }^{1}$.

Este estudo tem por objetivo avaliar instrumentos de mensuração da qualidade de vida (QLQ-C30 = Quality of Life Questionnaire Core (30 questões) e QLQ-H\&N35 = Quality of Life Questionnaire - Head and Neck Cancer Module (35 questões)) e seu potencial valor prognóstico nos pacientes com carcinoma epidermóide da cabeça e pescoço.

\section{MATERIAL E MÉTODO}

Foram incluídos prospectivamente 31 pacientes com carcinoma epidermóide de cabeça e pescoço atendidos no Departamento de Cirurgia de Cabeça e Pescoço e Otorrinolaringologia do Hospital Heliópolis, Hosphel, São Paulo, entre agosto de 1999 e novembro de 2000.

Em relação ao sítio primário, 12 pacientes apresentavam tumor de boca, 6 de laringe, 8 de orofaringe, 4 de hipofaringe e 1 de seio da face. Quanto ao estadiamento, 2 apresentavam doença em estádio I, 3 em estádio II, 9 em estádio III e 17 em estádio IV. O tratamento cirúrgico foi empregado em 26 pacientes, enquanto que 5 pacientes receberam tratamento radioterápico. Quanto ao grau de instrução, 2 pacientes eram analfabetos, $23 \mathrm{com}$ primeiro grau incompleto, $2 \mathrm{com}$ primeiro grau completo, $1 \mathrm{com}$ segundo grau incompleto e $3 \mathrm{com}$ segundo grau completo. Quanto ao sexo, 28 pacientes eram masculinos e 3 femininos.

$\mathrm{Na}$ avaliação da qualidade de vida, empregou-se o questionário QLQ-C30 versão 3, com a inclusão do módulo QLQ-H\&N35, ambos desenvolvidos pela EORTC - European Organization for Research and Treatment of Cancer. Os questionários foram aplicados pelo mesmo entrevistador para todos os pacientes, previamente ao tratamento.

O QLQ-C30 apresenta 30 questões agrupadas em 5 escalas funcionais, 9 escalas relacionadas a sintomas e uma escala global. O QLQ-H\&N35 apresenta 35 questões, das quais 30 são agrupadas em 13 escalas e 5 de resposta simples. As respostas foram convertidas em uma escala linear de pontuação, com valores de 0 a 100, conforme preconizado pela EORTC. Os resultados foram expressos em médias, com seus respectivos intervalos de confiança. Uma pontuação alta nas questões relacionadas aos sintomas reflete a presença mais intensa dos mesmos, enquanto uma pontuação alta nas questões relacionadas à função refletem a maior capacidade física do paciente.

Foram avaliadas as pontuações em relação aos diferentes sítios primários, estadiamento da doença e recidiva (ou persistência) nos primeiros 12 meses pós-tratamento. As diferenças entre os grupos foram aferidas pelo teste de Mann-Whitney, considerando um erro alfa igual ou inferior a $5 \%$.

\section{RESULTADOS}

A Tabela 1 apresenta a pontuação alcançada em cada escala dos questionários QLQ-C30 e QLQ-H\&N35 em relação ao estadiamento e controle da doença.

Foi observada diferença significativa na pontuação das escalas relacionadas à fadiga $(\mathrm{p}=0,03)$, qualidade de vida global $(\mathrm{p}=0,05)$ e dificuldade de comer em público $(\mathrm{p}=0,03)$ entre os pacientes com doença controlada e aqueles com recidiva nos primeiros 12 meses pós-tratamento. Considerando apenas estas três escalas, a taxa de recidiva (ou persistência) da doença entre os pacientes com pontuação desfavorável em pelo menos duas escalas foi de $90 \%(10 / 11)$, enquanto que $65 \%(13 / 20)$ daqueles com pontuação favorável em pelo menos duas escalas estavam com a doença controlada ao término de 12 meses.

\section{DISCUSSÃO}

Embora seja presumível que a doença em estádio avançado apresente mais sintomas, cause maior disfunção e conseqüentemente se relacione com pior qualidade de vida, isto pode ser verdadeiro para um indivíduo mas 
Tabela 1. Pontuação média dos questionários QLQ-C30 e QLQ-H\&N35

\begin{tabular}{|c|c|c|c|c|c|}
\hline \multirow[t]{2}{*}{ Escalas } & \multirow[t]{2}{*}{ Média $\left(\mathrm{IC}_{95 \%}\right)$} & \multicolumn{2}{|c|}{ Estádio } & \multicolumn{2}{|c|}{ Recidiva } \\
\hline & & | e || & III e IV & Não & Sim \\
\hline \multicolumn{6}{|l|}{ QLQ-C30 } \\
\hline Físico & $91(85-96)$ & 97 & 89 & 89 & 92 \\
\hline Emocional & 71 (62-79) & 80 & 69 & 68 & 73 \\
\hline Cognitivo & $85(78-91)$ & 96 & 82 & 83 & 86 \\
\hline Social & $86(79-94)$ & 90 & 86 & 89 & 84 \\
\hline Global * & $73(63-83)$ & 86 & 70 & 84 & 65 \\
\hline Dor & $26(14-37)$ & 0 & 31 & 19 & 31 \\
\hline Dispnéia & $5(0-12)$ & 6 & 5 & 2 & 7 \\
\hline Insônia & $31(17-44)$ & 13 & 34 & 25 & 35 \\
\hline Perda apetite & $20(9-30)$ & 6 & 22 & 20 & 19 \\
\hline Constipação & $14(4-24)$ & 6 & 16 & 23 & 7 \\
\hline Diarréia & 0 & 0 & 0 & 0 & 0 \\
\hline \multicolumn{6}{|l|}{ QLQ-H\&N35 } \\
\hline Olfato & $18(7-28)$ & 3 & 21 & 10 & 24 \\
\hline Fala & $26(16-36)$ & 11 & 29 & 31 & 22 \\
\hline Comer público * & $22(13-31)$ & 8 & 25 & 12 & 29 \\
\hline Contato social & $15(9-20)$ & 5 & 17 & 13 & 16 \\
\hline Sexualidade & $30(19-41)$ & 20 & 32 & 24 & 35 \\
\hline Dentição & $22(9-35)$ & 6 & 25 & 20 & 23 \\
\hline Abertura boca & $25(11-39)$ & 0 & 30 & 20 & 29 \\
\hline Boca Seca & $16(5-27)$ & 0 & 20 & 10 & 21 \\
\hline Saliva & 27 (16-39) & 6 & 32 & 17 & 35 \\
\hline Tosse & $22(11-32)$ & 6 & 25 & 20 & 23 \\
\hline Sentir doente & $16(6-26)$ & 6 & 18 & 12 & 19 \\
\hline
\end{tabular}

* variáveis significantes nos pacientes controlados ou recidivados após 12 meses por tratamento.

não reflete a realidade em um grupo heterogêneo de pacientes. Mesmo que uma relação entre o estadiamento e a qualidade de vida possa ser estabelecida em amostras maiores, não deve ser desprezada a grande variação individual, pois pacientes com doença em estádio semelhante podem valorizar diferentemente os seus sintomas e as suas limitações. Assim, para avaliar os efeitos de diferentes modalidades terapêuticas sobre a qualidade de vida dos pacientes, também deve ser avaliada a qualidade de vida prévia ao tratamento e a variação individual, pois a escala de pontuação é um ponto frágil dos questionários. O momento da avaliação também influencia os resultados, sendo observado um decréscimo da qualidade de vida imediatamente após o tratamento, com retorno gradual aos valores basais (pré-tratamento) após um intervalo de aproximadamente 6 meses. O controle da doença pode ocasionar uma melhora das funções emocionais, embora possa haver um decréscimo nas funções físicas ${ }^{2}$. As características das diferentes modalidades terapêuticas quanto às seqüelas e o tempo de reabilitação também devem ser consideradas.

Além da escala relacionada à fadiga, aquela relativa à dificuldade de comer em público e a de qualidade de vida global mostraram relação com a recidiva da doença nos primeiros 12 meses pós-tratamento. Com as limitações do estadiamento e da histopatologia em definir o prognóstico individual frente às modalidades terapêuticas disponíveis, os aspectos relacionados à qualidade de vida merecem uma maior investigação. A casuística estudada é heterogênea e seu tamanho não permite uma análise multivariada, por isto a interpretação destes achados exige cautela, porém a motivação para o tratamento e a presença de comorbidades não podem ser esquecidas no planejamento terapêutico.

A depressão é um achado freqüente em pacientes com câncer, embora nem sempre receba atenção por parte do oncologista ${ }^{3}$. Além de interferir na avaliação da qualidade de vida, a depressão pode afetar o sistema imunológico, favorecendo a recidiva da doença. A relação entre os fatores psicossociais e a progressão do câncer é uma área de recente interesse e, diante da complexidade do assunto, ainda não existem estudos conclusivos ${ }^{4,5}$.

No nosso meio, a maioria dos pacientes chegam ao especialista com doença em estádio avançado 
(estádios III e IV) e normalmente recebem tratamento combinado. O tempo despendido com o tratamento, considerando os exames prévios, o tempo de internação (cirurgia) e o tratamento radioterápico é de aproximadamente 2 a 3 meses. A morbidade do tratamento e suas seqüelas definitivas comprometem significativamente a qualidade de vida do paciente neste período. A reabilitação, especialmente quanto à fala e deglutição, também pode ser demorada, o que explica o longo intervalo para que o paciente recupere sua qualidade de vida. Uma recidiva precoce revela a limitação do médico ao indicar um tratamento com elevada morbidade, mesmo que este constitua a única (e pequena) oportunidade de cura. Portanto, a qualidade de vida também deve ser considerada entre os pacientes que não alcançam o controle da doença, avaliando a diferença entre a preservação de estruturas (preservar órgãos implica em preservar função) e uma cirurgia "higiênica".

O questionário pode ser auto-aplicado, mas a baixa escolaridade da maioria dos pacientes impôs a presença de um entrevistador, não possibilitando a aplicação dos questionários de forma uniforme mas adaptando-os aos diferentes níveis de compreensão, considerada a limitação intelectual do grupo. O questionário da EORTC foi formulado com o intuito de permitir a comparação entre diferentes populações. A semelhança da pontuação no presente estudo e a relatada em estudos conduzidos na Europa sugerem que o questionário tenha atingido este objetivo ${ }^{2,6}$, devendo entretanto, ser difundido o seu uso para um maior número de pacientes.

\section{CONCLUSÃO}

A qualidade de vida prévia ao tratamento pode constituir um indicador prognóstico relevante para os pacientes com câncer de cabeça e pescoço.

\section{REFERÊNCIAS BIBLIOGRÁFICAS}

1. Hammerlid E, Wirblad B, Sandin C. Malnutrition and food intake in relation to quality of life in head neck cancer patients. Head Neck 1998; 20:540-8.

2. Hammerlid E, Silander E, Hörnestam RN. Health-related quality of life three years after diagnosis of head and neck cancer - A longitudinal study. Head Neck 2001;23:113-25.

3. Bukberg J, Penman D, Holland JC. Depression in hospitalized cancer patients. Psychosom Med 1984;46:199-212.

4. De Graeff A, De Leeuw JRJ, Ros WJG. Pretreatment factors predicting quality of life after treatment for head and neck cancer. Head Neck 2000;22:398-407.

5. Garssen B, Goodkin K. On the role of immunological factors as mediators between psychosocial factors and cancer progression. Psychiatry Res 1999;85:51-61.

6. Aaronson NK, Ahmedzai S, Bergman B. The European Organization for Research and Treatment of Cancer QLQ-C30: a quality of life instrument for use in international clinical trials. J Natl Cancer Inst 1993;85:365-76 